Y. Shikata

Nagoya Math. J.

Vol. 38 (1970), 53-61

\title{
ON A HOMOLOGY THEORY ASSOGIATED TO FOLIATIONS
}

\author{
YOSHIHIRO SHIKATA
}

Dedicated to Professor Katuzi Ono on his 60th birthday

\section{Introduction.}

In this note, we associate a foliated singular homology theory to a foliation on a compact manifold $X$ and construct a spectral sequence which relates the foliated homology to the ordinary homology of $X$. Since the foliated homology is so constructed as to be related closely to a certain topological behavior of the foliation, we may expect that further study of the spectral sequence reveals some information on topology of foliation. The study and applications will be given in a sequel.

\section{A double complex associated to a plane field.}

Let $X$ be a compact smooth manifold having a plane field $\Pi$. A smooth map $\alpha$ of $(i+j)$-cube $I^{i} \times I^{j}$ into $X$ is called a singular $(i, j)$-cube on $(X ; \Pi)$, if the map $\alpha$ satisfies that

(i) for any $Q \in I^{j}$, the restriction of $\alpha$ to the $i$-plane $I^{i} \times Q$ of $I^{i} \times I^{j}$ is tangent to the plane field, that is,

$$
d \alpha\rfloor_{I^{i} \times Q}\left(T_{p}\left(I^{i}\right)\right) \subset \Pi_{\alpha(P \times Q)}, \quad\left(\text { for all } P \in I^{i}\right),
$$

(ii) for any $P \in I^{i}$, the restriction of $\alpha$ to $j$-plane $P \times I^{j}$ is transversal to the plane field, that is,

$$
\left.d \alpha\right|_{P \times I^{j}}\left(T_{Q}\left(I^{j}\right)\right) \cap \Pi_{\alpha(P \times Q)}=0 \quad\left(\text { for all } Q \in I^{j}\right) .
$$

Denote by $C_{i, j}^{\prime}(X, \Pi)$ the free abelian group generated over all the $(i, j)$-cubes and define face operators $F_{k ;}^{\varepsilon}, F_{; k}^{\varepsilon}(\varepsilon=0,1)$ as to be the linear maps satisfying that

Received March 31, 1969 


$$
\begin{aligned}
& \left(F_{; k}{ }^{\varepsilon} \alpha\right)\left(s_{1} \cdots s_{i}, t_{1} \cdots t_{j}\right)=\alpha\left(s_{1} \cdots s_{k-1}, \varepsilon, s_{k} \cdots s_{i}, t_{1} \cdots t_{j}\right) \\
& \left(F_{; k}^{\varepsilon} \alpha\right)\left(s_{1} \cdots s_{i}, t_{1} \cdots t_{j}\right)=\alpha\left(s_{1} \cdots s_{i}, t_{1} \cdots t_{k-1}, \quad \varepsilon, \quad t_{k} \cdots t_{j}\right) .
\end{aligned}
$$

Define operators $\xi=\xi_{i, j}, \eta=\eta_{i, j}$ on $C_{i, j}^{\prime}$ by

$$
\xi=\sum_{k=1}^{i}(-1)^{k}\left(F_{k ;}{ }^{1}-F_{k ;}{ }^{0}\right), \quad \eta=(-1)^{i} \sum_{k=1}^{j}(-1)^{k}\left(F_{; k}{ }^{1}-F_{; k}{ }^{0}\right),
$$

then $\xi, \eta$ satisfy that

$$
\xi \circ \xi=\eta \circ \eta=0, \xi \circ \eta+\eta \circ \xi=0 .
$$

Let $D_{k \text {; }}\left(\right.$ resp. $\left.D_{; j k}\right)$ be linear maps of $C_{i-1, j}^{\prime}$ into $C_{i, j}^{\prime}$ (resp. $C_{i, j-1}^{\prime} \longrightarrow C_{i, j}^{\prime}$ ) such that

$$
\begin{aligned}
& \left(D_{k ;} \alpha\right)\left(s_{1} \cdots s_{i}, t_{1} \cdots t_{j}\right)=\alpha\left(s_{1} \cdots s_{k-1}, s_{k+1} \cdots s_{i}, t_{1} \cdots t_{j}\right) \\
& \left(D_{; k} \alpha\right)\left(s_{1} \cdots s_{i}, t_{1} \cdots t_{j}\right)=\alpha\left(s_{1} \cdots s_{i}, t_{1} \cdots t_{k-1}, t_{k+1} \cdots t_{j}\right)
\end{aligned}
$$

then it is easy to see that

$$
\begin{aligned}
& D_{k ;} \circ D_{m ;}=D_{m+1 ;} \circ D_{k ;}, D_{; k} \circ D_{; m}=D_{; m+1} \circ D_{; k}(k \leqq m), \\
& F_{k ;}^{\varepsilon} \circ D_{m ;}=\left\{\begin{array}{cc}
D_{m-1 ;} \circ F_{k ;}^{\varepsilon}(k<m), \quad F_{; k}^{\varepsilon} \circ D_{; m} \\
1 \quad(k=m), \\
D_{m ;} \circ F_{k-1 ;}^{\varepsilon}(k>m),
\end{array}=\left\{\begin{array}{cc}
D_{; m-1} \circ F_{; k}^{\varepsilon} & (k<m) \\
1 & (k=m) \\
E_{; m} \circ F_{; k-1}^{\varepsilon} & (k>m) .
\end{array}\right.\right.
\end{aligned}
$$

Therefore the quotient $C_{i, j}$ of $C_{i, j}^{\prime}$ given by

$$
C_{i, j}=C_{i, j}^{\prime} / \sum_{k=1}^{i-1} D_{k ;} C_{i-1, j}^{\prime}+\sum_{k=1}^{j-1} D_{; k} C_{i, j-1}^{\prime}
$$

turns out to be a double complex having boundary operators $\xi, \eta$. Denote by $\left(S_{n}(X), \partial\right)$ the (normalized) cubical singualr chain complex of $X$, then the single complex $C_{n}=\sum_{i+j=n} C_{i, j}$ is naturally imbedded into $S_{n}(X)$ as a subcomplex because the operators $\xi, \eta$ and $\hat{o}$ satisfy that

$$
\xi+\eta=\hat{o} .
$$

Lemma 1. Let $N$ be an integer and let $e_{p, q}$ be a map of $I^{i} \times I^{j}$ into $I^{i-1} \times$ $[p / N, p+1 / N] \times I^{i-1} \times[q / N, q+1 / N]$ given by

$$
e_{p, q}\left(s_{1} \cdots s_{i}, t_{1} \cdots t_{j}\right)=\left(s_{1} \cdots s_{i-1}, p+s_{i} / N, t_{1} \cdots t_{j-1}, q+t_{j} / N\right) .
$$

then for any $\alpha \in C_{i, j}^{\prime}$ there exists $f(\alpha) \in C_{i+1, j}^{\prime}+C_{i, j+1}^{\prime}$ such that

$$
(\xi+\eta) f(\alpha)+f((\xi+\eta) \alpha)=\alpha-\sum_{p, q} \alpha \circ e_{p, q} \text { in } C_{i, j} .
$$


For a chain $\gamma=\sum c_{k} \alpha_{k}\left(c_{i} \neq 0\right)$ of $C_{i, j}^{\prime}$, define $\operatorname{Car}(\gamma)$ to be $\underset{k}{\cup} \alpha_{k}\left(I^{i} \times I^{j}\right)$ and for an open set $U$ in $X$ let

$$
\begin{aligned}
& C_{i, j}^{\prime}{ }^{U}=\left\{\gamma \in C_{i, j}^{\prime} / \operatorname{Car}(\gamma) \subset U\right\}, \\
& C_{i, j}{ }^{U}=C_{i, j}^{\prime} U / \sum_{k=1}^{i=1} D_{k ;} C_{i-1, j}^{\prime} U+\sum_{k=1}^{j-1} D_{; j k} C_{i, j-1}^{\prime} U, \\
& C_{n}{ }^{U}=\sum_{i+j=n} C_{i, j}{ }^{U} .
\end{aligned}
$$

Proposition 1. Let $\{U\}$ be an open covering of $X$ and let $C_{n}{ }_{n}^{\{U\}}$ be the union of $C_{n}{ }^{U}, U \in\{U\}$. Then the inclusion $C_{n}{ }_{n}(U\}$ into $C_{n}$ is a chain equivalence.

A plane field $\Pi$ on $X$ is said to be completely integrable if there is a foliation on $X$ such that for any $x \in X, \Pi_{x}$ agrees with the tangent plane at $x$ of the leaf through $x$.

Lemma 2. Suppose that the plane field $\Pi$ on $X$ is completely integrable then for any open set $U$ of $X$ and for any point $x \in U$, there is a neighbourhood $V$ of $x$ such that $V \subset U$ and $C_{n}{ }^{V}$ is acyclic.

Proof. Take a flat coordinate $\left(x_{1} \cdots x_{p}, y_{1} \cdots y_{q}\right)\left(-\varepsilon<x_{i}, y_{i}<\varepsilon\right)$ in a neighbourhood $V$ of $x$ so that $p$-planes defined by $\left\{y_{i}=\right.$ const. $\}$ form (locally) the leaves of the foliation around $x$. Then the homotopy $h_{t}^{1}$ in $V$ defined by

$$
h_{t}^{1}\left(x_{1} \cdots x_{p}, y_{1} \cdots y_{q}\right)=\left(t x_{1} \cdots t x_{p}, y_{1} \cdots y_{q}\right)
$$

gives a chain homotopy in $C_{n}^{\prime V}$ between $\alpha \in C_{i, j}^{\prime}{ }^{V}$ and a chain $\pi^{1}(\alpha) \in C_{0 ; j}^{\prime}{ }^{v}$, also the homotopy $h_{t}^{2}$ defined by

$$
h_{t}{ }^{2}\left(0 \cdots 0, y_{1} \cdots y_{q}\right)=\left(0 \cdots 0, t y_{1} \cdots t y_{q}\right)
$$

gives a chain homotopy in $C_{n}^{\prime V}$ between $\pi^{1} \alpha \in C_{0, j}^{\prime}{ }^{V}$ and $\pi^{2}\left(\pi^{1} \alpha\right) \in C_{0,0}^{\prime}{ }^{V}$.

Thus, following a standard method of homology theory of (co-)sheaves ([1] [4]), we have

Proposition 2. If the plane field $\Pi$ on $X$ is completely integrable then the Čech homology group $H_{*}(X ; G)$ is isomorphic to the projective limit of the homology groups $H_{*}\left(C_{n}{ }^{\{U\}} ; G\right)$ for any module $G$.

Therefore combining the propositions 1 and 2, we have

THEOREM 1. If the plane field $\Pi$ on a compact manifold $X$ is completely integrable, then for any module $G$. It holds that

$$
H_{*}(X ; G)=H_{*}\left(C_{n} \otimes G, \xi+\eta\right) .
$$




\section{A spectral sequence associated to $\mathrm{C}_{i, j}$.}

Introduce two regular filtrations $F^{\mathrm{I}}$ and $F^{\mathbb{I}}$ into $C_{i, j}$ as follows:

$$
F_{p}^{\mathrm{I}} C=\sum_{r \leqq p} \sum_{q} C_{r, q}, F_{q}^{\mathbb{\sharp}} C=\sum_{s \leqq q} \sum_{p} C_{p, s} .
$$

Then there result filtrations of $H_{*}(C, \xi+\eta)$ and two spectral sequences $\mathrm{I}_{i, j}^{r}$ and $\Pi_{i, j}^{r}$ ([2]), we investigate several terms of the spectral sequences in case that II is completely integrable.

\section{1 On terms $\Pi_{i, j}^{1}$.}

It is known ([2]) that

$$
\mathrm{I}_{i, j}^{1}=H\left(C_{i, j}, \eta\right), \quad \Pi_{i, j}^{1}=H\left(C_{i, j}, \xi\right) \text {. }
$$

On the other hand, we easily have

Lemma 3. If the plane field II is completely integrable, then the chain complex $\left(C_{i, 0}, \xi\right)$ is identified with the chain complex $\left(\sum_{L} S_{i}(L), \partial\right)$, where the sum $\sum_{L}$ is taken over all the leaves of the foliation.

The lemma 3 above obviously yields

Proposition 3. If the plane field $\Pi$ is completely integrable, then it holds that

$$
\text { II }_{i, 0}^{1}=\sum_{L} H_{i}(L ; Z) \text {, }
$$

where the sum $\sum_{L}$ is taken over all the leaves of the foliation.

Lemma 4. If the plane field $\Pi$ is completely integrable and if an $(i, j)$-cube $\alpha$ is non zero in $C_{i, j}^{\prime} / \sum_{k} D_{; k} C_{i, j-1}^{\prime}$, then the first $i$-faces $F_{k ;}{ }^{\varepsilon} \alpha(\varepsilon=0,1)$ are non zero in $C_{i-1, j}^{\prime} / \sum_{k} D_{; k} C_{i-1, j-1}^{\prime}$.

Proof. Suppose on the contrary that $F_{k ;}{ }^{8} \alpha$ is independent of the coordinate $t_{1}$ of $I^{j}$ and let $Q(x)$ denote the point $\left(x, t_{2} \cdots t_{n}\right)$ of $I^{j}$, then for any $x \in I, \alpha\left(I^{i} \times Q(x)\right)$ lies in the same leaf $L$, because they have a point $\left.\alpha\left(s_{1} \cdots s_{i}, x, t_{2} \cdots t_{j}\right)\right)$ in common, therefore the vector $\partial / \partial x=\partial / \partial t_{1}$ is mapped by $d \alpha$ into the tangent plane of $L$, thus we have that $\partial \alpha / \partial t_{1}=0$ at every point of $I^{i} \times I^{j}$ and conclude that $\alpha$ itself is independent of $t_{1}$.

Lemma 5. If the plane field $\Pi$ is completely integrable, then for each chain $\gamma \in C_{i, j}$, there corresponds a chain $\gamma^{\prime} \in C_{i, j}^{\prime}$, such that $\gamma^{\prime}=\gamma$ in $C_{i, j}$ and satisfying 
(i)

$$
\xi \gamma=0 \text { in } C_{i, j} \text { implies } \xi \gamma^{\prime}=0 \text { in } C_{i, j}^{\prime} / \sum_{k} D_{k ;} C_{i-1, j}^{\prime} \text {, }
$$

$$
\xi \gamma=\gamma_{2} \text { in } C_{i, j} \text { implies } \xi \gamma_{1}^{\prime}=\gamma_{2}^{\prime} \text { in } C_{i, j}^{\prime} / \sum_{k} D_{k ;} C_{i-1, j}^{\prime} \text {. }
$$

Proof. Let $V_{i, j}$ be a set of all the $(i, j)$-cubes which are indepent of $t_{k}$ for some $1 \leqq k \leqq j$ and let $W_{i, j}$ be the set of all the $(i, j)$-cubes which are not in $V_{i, j}$. Then $C_{i, j}^{\prime}$ decomposes into a direct sum of free groups $A_{i, j}$ and $B_{i, j}$ generated over $V_{i, j}$ and $W_{i, j}$ respectively. Denote by $x^{\prime}$ and $x^{\prime \prime}$ the $A_{i, j}$ and $B_{i, j}$ component of $x \in C_{i, j}^{\prime}$ respectively, then Lemma 4 yields that

$$
(\xi x)^{\prime \prime}=\xi\left(x^{\prime \prime}\right) \text { for } x \in C_{i, j}^{\prime} .
$$

Now choose a representative $\tilde{\gamma} \in C_{i, j}^{\prime}$ for $\gamma \in C_{i, j}$ and define $\gamma^{\prime}$ by $r^{\prime}=(\tilde{\gamma})^{\prime}$. Suppose that

$$
\xi \gamma=0 \text { in } C_{i, j}
$$

then

$$
\xi \tilde{\gamma}=x \text { for some } x \in \sum_{k} D_{k ;} C_{i-2, j}^{\prime},+\sum_{k} D_{; k} C_{i-1, j-1}^{\prime} .
$$

Thus we have that

$$
\xi\left(\gamma^{\prime}\right)=\xi\left(\tilde{\gamma}-(\tilde{\gamma})^{\prime \prime}\right)=\xi \tilde{\gamma}-(\xi \tilde{\gamma})^{\prime \prime}=x-x^{\prime \prime},
$$

therefore we see that

$$
\xi\left(\gamma^{\prime}\right)=0 \text { in } C_{i-1, j}^{\prime} / \sum D_{k ;} C_{i-2, j}^{\prime},
$$

and we also see (ii) as in the same way above.

Define a linear $\operatorname{map} F_{; Q}\left(Q \in I_{j}\right)$ of $C_{i, j}^{\prime}$ into $C_{i, 0}^{\prime}$ by

$$
\left(F_{; Q^{\alpha}} \alpha\right)\left(s_{1} \cdots s_{i}\right)=\alpha\left(s_{1} \cdots s_{i}, t_{1}(Q) \cdots t_{j}(Q)\right),
$$

then we easily see that $F_{; Q}$ commutes with $F_{k ;}$ and $D_{k ;}$ for any $1 \leqq k \leqq i$. Thus Lemma 5 yields

PROPOSITION 4. If the plane field $\Pi$ is completely integrable, then for each chain $r \in C_{i, j}$ there exists a representative $\gamma^{\prime} \in C_{i, j}^{\prime}$ satisfying

(i) $\quad \xi \gamma=0$ in $C_{i, j}$ implies $\xi\left(F_{; Q} \gamma^{\prime}\right)=0$ in $C_{i, 0}$,

(ii) $\quad \xi \gamma_{1}=\gamma_{2}$ in $C_{i, j}$ implies $\xi\left(F_{; Q} \gamma_{1}^{\prime}\right)=F_{; Q} \gamma_{2}^{\prime}$ in $C_{i, 0}$.

In the other words, $\gamma \in C_{i, j}$ is a $\xi$-cycle only if there is a $j$-dimensional 
family of $\partial$-cycles of $\sum_{L} S_{i}(L)$ and a $\xi$-cycle $\gamma_{1}$ is a $\xi$-boundary only if every $\partial$-cycle of the family is a $\partial$-boundary of $\sum_{L} S_{i+1}(L)$.

\section{2 On terms $\Pi_{i, j}^{2}$}

Since $\eta$ commutes with $\xi, \eta$ defines canonically a homomorphism of $H\left(C_{i, j}, \xi\right)$ into $H\left(C_{i, j-1}, \xi\right)$ which is denoted also by $\eta$. Then it is known ([2]) that

$$
\Pi_{i, j}^{2}=H\left(H\left(C_{i, j} \xi\right), \eta\right) \text {. }
$$

Therefore we deduce from Proposition 3 that

Proposition 5. If the plane field $\Pi$ is completely integrable, then it holds that

$$
\mathrm{II}_{i, 0}^{2}=\sum_{L} H_{i}(L ; Z) / \eta\left(H\left(C_{i, 1}, \xi\right),\right.
$$

where the sum $\sum_{L}$ is taken over all the leaves of the foliation.

DeFinition. In case that the plane fields $\Pi$ is derived from a foliation, we call the terms $\Pi_{i, 0}^{1}$ and $\Pi_{i, 0}^{2}$ the $i$-th homology groups of the foliation of the first and second kind, respectively, and we write

$$
{ }^{1} H_{i}(\Pi)=\Pi_{i, 0}^{1}, \quad{ }^{2} H_{i}(\Pi)=\Pi_{i, 0}^{2} .
$$

Roughly speaking, for $p=\operatorname{dim} \Pi$, the dimension of free part of $I I_{p, 0}^{1}$ represents the number of oriented closed leaves of the foliation and that of $I I_{p, 0}^{2}$ corresponds to the number of oriented closed leaves which can not be connected each other by any 1-dimensioned family of oriented closed leaves.

\section{3 On terms $\Pi_{i, j}^{\infty}$}

Since it is known ([2]) that

$$
\sum_{i+j=n} \Pi_{i, j}^{\infty}=\mathscr{G} H\left(C_{n}, \xi+\eta\right),
$$

we see that Theorem 1 implies that

Proposition 6. If the plane field $\Pi$ is completely integrable, then it holds that

$$
\sum_{i+j=n} \Pi_{i, j}^{\infty}=\mathscr{G} H_{n}(X ; Z) .
$$

\section{Remarks for dually foliated structures.}

A pair of plane fields $\left(\Pi_{1}, \Pi_{2}\right)$ on a compact manifold $X$ is called an orthogonal splitting of the tangent bundle $T(X)$ if it holds that 


$$
\Pi_{1} \perp \Pi_{2}, \quad \Pi_{1}+\Pi_{2}=T(X) .
$$

Let $\left(X ; \Pi_{1}, \Pi_{2}\right)$ be a compact manifold with an orthogonal splitting $\left(\Pi_{1}, \Pi_{2}\right)$. A smooth map $\alpha$ of $(i+j)$-cube $I^{i} \times I^{j}$ into $X$ is said to be a singular $(i, j)$-cube of $\left(X ; \Pi_{1}, \Pi_{2}\right)$ if the map $\alpha$ satisfies that

(i) for any $Q \in I^{j}$, the restriction of $\alpha$ to the $i$-plane $I^{i} \times Q$ of $I^{i} \times I^{j}$ is tangent to the plane field $\Pi_{1}$.

(ii) for any $P \in I^{i}$, the restriction $\alpha$ to the $j$-plane $P \times I^{j}$ of $I^{i} \times I^{j}$ is tangent to the plane field $\Pi_{2}$.

The free abelian group $\Gamma_{i, j}^{\prime}$ generated over all the $(i, j)$-cubes of $\left(X ; \Pi_{1}, \Pi_{2}\right)$ has face operators $F_{; k}, F_{k}$; and degeneracy operators $D_{; k}, D_{k}$; defined in the same way as in $\$ 1$. Therefore $\Gamma_{i, j}^{\prime}$ and the quotient

$$
\Gamma_{i, j}=\Gamma_{i, j}^{\prime} / \sum D_{k ;} \Gamma_{i-1, j}^{\prime}+\sum D_{; k} \Gamma_{i, j-1}^{\prime}
$$

turn out to be double complexes with boundary operators $\xi, \eta$ given by

$$
\xi=\sum(-1)^{k}\left(F_{k ;}^{1}-F_{k ;}^{0}\right), \quad \eta=\sum(-1)^{k+i}\left(F_{; k}^{1}-F_{; k}^{0}\right) .
$$

An orthogonal splitting $\left(\Pi_{1}, \Pi_{2}\right)$ of $T(X)$ is said to be completely integrable if the plane fields $\Pi_{i}(i=1,2)$ are completely integrable by foliations $\left(F_{i}\right)(i=1,2)$ on $X$, and if each point $x \in X$ has a coordinate neighbourhood $U\left(x_{1} \cdots x_{p}, y_{1} \cdots y_{q}\right)$ such that the $p$-plane given by $y_{i}=$ const. is a leaf of $\left(F_{1}\right)$ in $U$ and the $q$-plane given by $x_{i}=$ const. is a leaf of $\left(F_{2}\right)$ in $U$. The following theorem is proved easily as in the same way in $\$ 1$;

TheOREM 2. If an orthogonal splitting $\left(\Pi_{1}, \Pi_{2}\right)$ on a compact manifold $X$ is completely integrable, then for the single complex $\Gamma_{n}=\sum_{i+j=n} \Gamma_{i, j}$, it holds that

$$
H_{*}(X ; Z)=H_{*}\left(\Gamma_{n}, \xi+\eta\right) \text {. }
$$

Therefore as in $\S 2$ the spectral sequence $\left(E_{i, j}^{r}\right)$ associated to the filtration

$$
F_{q}=\sum_{s \leqq q} \sum_{p} \Gamma_{p, s}
$$

relates $E_{i, j}^{2}=H\left(H\left(\Gamma_{i, j}, \xi\right), \eta\right)$ to $H_{*}(X ; Z)$.

Since $C_{i, 0}=\Gamma_{i, 0}$ we obviously have

LEMma 6. If an orthogonal splitting $\left(\Pi_{1}, \Pi_{2}\right)$ is completely integrable, then it holds that 


$$
{ }^{1} H_{i}\left(\Pi_{1}\right)=E_{i, 0}^{1} \text {. }
$$

Define linear maps $F_{; Q}$ and $F_{P ;}\left(Q \in I_{j}, P \in I_{i}\right)$ of $\Gamma_{i, j}^{\prime}$ into $\Gamma_{i, 0}^{\prime}$ and $\Gamma_{0, j}^{\prime}$, respectively, by

$$
\begin{aligned}
& \left(F_{; Q} \alpha\right)\left(s_{1} \cdots s_{i}\right)=\alpha\left(s_{1} \cdots s_{i}, t_{1}(Q) \cdots t_{j}(Q)\right) \\
& \left(F_{P ;} \alpha\right)\left(t_{1} \cdots t_{j}\right)=\alpha\left(s_{1}(P) \cdots s_{i}(P), t_{1} \cdots t_{j}\right) .
\end{aligned}
$$

Then we have the following proposition of which proof is quite similar to that of Proposition 4.

Proposition 7. If an orthogonal splitting $\left(\Pi_{1}, \Pi_{2}\right)$ is completely integrable, then for each chain $\gamma \in \Gamma_{i, j}$ there exists a representative $\gamma^{\prime} \in \Gamma_{i, j}^{\prime}$ such that

$$
\xi \gamma=0 \text { in } \Gamma_{i, j} \text { implies } \xi\left(F_{; Q} \gamma^{\prime}\right)=0 \text { in } \Gamma_{i, 0}
$$

(i) -2

$$
\eta \gamma=0 \text { in } \Gamma_{i, j} \text { implies } \eta\left(F_{P_{i}} \gamma^{\prime}\right)=0 \text { in } \Gamma_{0, j}
$$

$$
\xi \gamma_{1}=\gamma_{2} \text { in } \Gamma_{i, j} \text { implies } \xi\left(F_{; Q} \gamma_{1}^{1}\right)=F_{; Q} \gamma_{2}^{\prime} \text { in } \Gamma_{i, 0}
$$

$$
\eta \gamma_{1}=\gamma_{2} \text { in } \Gamma_{i, j} \text { implies } \eta\left(F_{P ;} \gamma_{1}^{\prime}\right)=F_{P ;} \gamma_{2}^{\prime} \text { in } \Gamma_{0, j} \text {. }
$$

Corollary 1. Let $\left(\Pi_{1}, \Pi_{2}\right)$ be a completely integrable splitting of $T(X)$, if there exists non trivial $\xi$-cycle $r \in \Gamma_{i, j}$ or $\eta$-cycle $r \in \Gamma_{i, j}$, then there exists $a j$ dimensional family of $\xi$-cycles $\gamma_{Q} \in \Gamma^{\prime}{ }_{i, 0}\left(Q \in I^{j}\right)$ or an $i$-dimensional family of $\eta$ cycles $\gamma_{P} \in \Gamma^{\prime}{ }_{0, j}\left(P \in I^{i}\right)$, respectively.

Thus we see that for a completely integrable splitting, if there is a non trivial $\eta$-cycle $x \in \Gamma_{0, j}$ of the form

$$
x=\xi y+\eta z, \quad y \in \Gamma_{1, j}, \quad z \in \Gamma_{0, j+1},
$$

then there is an 1-dimensional family of $\eta$-cycles $x_{P} \in \Gamma_{0, j}^{\prime}(P \in I)$ or a $(j-1)$-dimensional family of $\xi$-cycles $x_{Q} \in \Gamma_{1,0}^{\prime} \quad\left(Q \in I^{j-1}\right)$.

Now assume that $X$ is a 3-dimensional compact manifold for which

$$
H_{2}(X ; Z)=0
$$

and that $T(X)$ splits into mutually orthogonal 1-plane field $\Pi_{1}$ and 2-plane field $\Pi_{2}$ such that the pair $\left(\Pi_{1}, \Pi_{2}\right)$ is completely integrable. Then if there is a non trivial $\eta$-cycle $x \in \Gamma_{0,2}$, an easy compution of the spectral sequence $\left(E_{i, j}^{r}\right)$ shows that there exists at least one non trivial $\xi$-cycle $y \in \Gamma_{1,0}^{\cdot}$ or a 1-dimensional family of $\eta$-cycles $z_{p} \in \Gamma_{0,2}^{\prime}$. Thus we have the following theorem as an elementary application of the spectral sequence. 
THEOREM 3. Let $X$ be a compact 3-manifold having a 2-dimensional foliation $\left(F_{2}\right)$ and let $\left(F_{1}\right)$ be the 1-dimensional foliation defined by the 1-plane field orthogonal to $\left(F_{2}\right)$. Then, if $\left(F_{2}\right)$ has only isolated closed leaves and if 2-dimensional integral homology of $X$ vanishes, there should be at least one closed orbit in $\left(F_{1}\right)$.

\section{BIBLIOGRAPHY}

[1] G. Bredon, Cosheaves and homology, Pacific J. of Math. Vol. 25, (1968), 1-32.

[2] H. Cartan and S. Eilenberg, Homological algebra, Princeton Univ. Press, (1956).

[ 3 ] G. Reeb, Sur les espaces fibrés et les varietes feuilletées, Actualités scientifiques et industrielles, Hermann Paris, $n^{\circ} 1183$.

[4] E. Spanier, Algebraic topology, McGraw-Hill, (1966).

Mathematical Institute,

Nagoya University 